\title{
Parameter Optimization of Real-Time RK Formula Based on Genetic Algorithm
}

\author{
Zhenquan Huang ${ }^{\mathrm{a}}$, Erke Tong ${ }^{\mathrm{b} *}$ and Suying Liao \\ Air Force Airborne Academy, Guilin 541003, China \\ ahuangzhenquan@163.com, b*oakey@sina.com \\ "The corresponding author
}

\begin{abstract}
Generally real-time Runge-Kutta (RK) formula should be provided with large stability region and small truncation error, when using real-time RK formula for real-time digital simulation of dynamics system. This paper analyses the stability region and truncation error of RK formula and considers the advantages of genetic algorithm for constrained optimization. And the parameter optimization for stability region and truncation error of RK formula is presented based on genetic algorithm principle and flow diagrams. Further, the maximum absolute stability region area and least truncation error of ODE23 and ODE34 and ODE45 RK formula can be calculated through setting nondeterministic parameters of RK formula as the optimization variables and setting eigenvalue stability region and truncation error as objective function. The parameter values of real-time RK formula are also obtained. Finally, this paper deduces the all order real-time RK formulas.
\end{abstract}

Keywords: Digital simulation; Genetic algorithm; Runge-Kutta formula; Real-Time simulation; Parameter optimization

\section{基于遗传算法的实时 RK 公式参数优化}

\author{
黄振全 ${ }^{1}$ 全二克 ${ }^{2}$ 廖素英 ${ }^{3}$ \\ (空军空降兵学院, 广西 桂林 541003 )
}

摘要: 在使用实时 Runge-Kutta ( RK) 公式对动力学系统进行实时数字仿真时, 一般要求实时 RK 公式具有大 稳定域和小截断误差。在研究遗传算法基本原理和优化流程图的基础上, 针对现有实时 RK公式的稳定域和截断误 差进行分析, 结合遗传算法求解约束优化问题的优点, 对实时 RK 公式的稳定域参数和截断误差参数进行了优化。 以实时 RK 公式的非确定参数为优化变量, 以关心的特征值稳定域面积和截断误差为目标函数的全局优化问题, 计 算得出实时三级二阶、四级三阶和五级四阶 RK 公式的最大绝对稳定域面积和此前提下的最小截断误差, 并且还得 出了各阶实时 $\mathrm{RK}$ 公式参数的具体数值, 最后推导出各阶实时 RK 公式。

关键词: 数字仿真; 遗传算法; 龙格-库塔公式; 实时仿真; 参数优化

中图分类号: 0241.81 文摘标识码: A

引言

实时 RK 公式是一种典型的实时数字仿真算法公式, 被广泛应用于动力学系统实时数字仿真 中。由于实时 $\mathrm{RK}$ 公式是一种近似积分方法, 在反复的递推运算中会引进误差, 为条件稳定, 对 
于已知数学模型为:

$$
\dot{y}(t)=f[t, y(t), u(t)] \quad y\left(t_{0}\right)=y_{0}
$$

的稳定的实时仿真系统, 当采用实时 RK 公式进行实时数字仿真计算时, 数值解的稳定性必须保 证, 误差必须在允许范围内, 否则计算结果将失去真实意义。由于 RK 的一般公式都是相同的, 但参数的不同选择使得稳定域和截断误差的大小不同, 对实时 RK 公式的适用范围有着极大的影 响。因此，对实时 RK 公式的参数进行优化有着重要的理论意义和实用价值。

相同的寻优问题，不同的优化算法有不同的寻优特性。单纯形法、梯度法、以及蚁群算法等, 这些方法都具有良好的寻优特性, 但也有各自的缺点, 如单纯形法对初值比较敏感, 容易陷入局 部最优解, 造成寻优失败; 梯度法要求目标函数具有一阶导数, 当目标函数具有窄长形的谷值时, 这种方法的效率很低, 甚至得不到优化解; 蚁群算法对于初始点的选取较为敏感, 波动较大, 算 法不够成熟。而本文采用的遗传算法是一种寻求全局最优且不需任何初始信息的高效优化方法, 从而对问题的依赖性较小，并且遗传算法对待寻优的函数基本无限制。具有简单通用、鲁棒性强、 适用于并行处理及高效实用等显著特点, 适合对实时 RK 公式的参数进行求解约束的优化问题。

\section{1 遗传算法}

\section{1 遗传算法基本原理}

遗传算法(GA)最先由 John Holland 于 1975 年提出, 它的思想源于生物遗传学和适者生存的自 然规律。遗传算法是一种基于生物自然选择与遗传机理的随机搜索算法。遗传算法抽象了生物体 的进化过程, 通过全面模拟自然选择和遗传机制, 以编码空间代替问题的参数空间, 以适应度函 数为评价依据, 以编码群体为进化基础, 以对群体中个体位串的遗传操作按所选择的适应值函数 通过复制、交叉及变异, 对个体进行篎选, 实现选择和遗传机制, 建立起一个迭代过程。在这一 过程中，通过随机重组编码位串中重要的基因，使新一代的位串集合优于老一代的位串集合，群 体的个体不断进化，直到满足一定的条件，逐渐接近最优解，最终达到求解问题的目的 ${ }^{[2,3]}$ 。

$\mathrm{GA}$ 的基本操作为：复制、交叉、变异。

(1)复制。复制是从一个旧的种群中选择生命力强的个体串产生新的种群的过程。根据串结构 的适配值拷贝，也就是指具有高适配值的串可能在下一代中产生一个或多个子孙。它模仿了自然 现象，应用了达尔文的适者生存理论。

(2)交叉。复制操作能从旧种群中选择出优秀者, 但是不能创造出新的染色体。而交叉模拟了 生物进化过程的繁殖现象, 通过两个染色体的交换组合来产生新的优良品种, 它的过程为: 在匹 配池中任选两个染色体，随机选择一点或多点交换点位置；交换双亲染色体交换点右边的部分， 即可得到两个新的染色体串，交叉体现了自然界中信息交换的思想。

(3)变异。变异运算来自生物在自然界的遗传环境中由于各种偶然因素引起的基因突变。首先 
在群体中随机选择一个个体, 对于选中的个体以一定的概率随机的改变串结构数据中某个串的值, 如: 它随机的将串中的某个数据由 1 变成 0 , 或由 0 变成 1 。同生物界一样, GA 中变异发生的概 率很低, 通常取值在 0.001 0.1 之间, 变异为新个体的产生提供了机会。如只有复制和交叉而没有 变异, 则无法在初始基因组合以外的空间进行搜索, 使得进化过程在早期就陷入局部最优解而进 入终止过程, 所以为了在尽可能大的空间中获得优化解, 变异操作是必需的 ${ }^{[4]}$ 。

\section{2 遗传算法优化流程图}

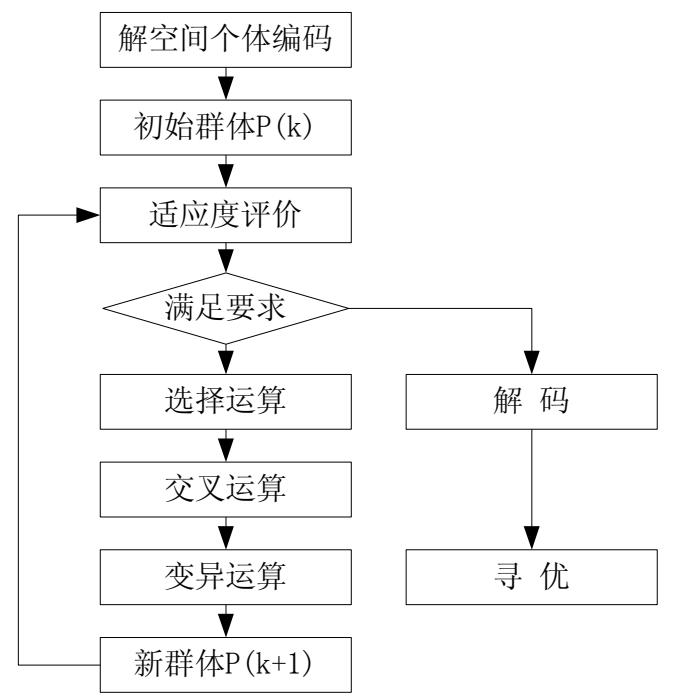

图 1 遗传算法优化流程图

\section{2 基于遗传算法的实时 RK 公式参数优化}

\section{1 实时 RK 公式稳定域参数优化}

由于实时 $R K$ 仿真算法是一种近似方法, 运用实时 RK 算法公式进行实时仿真, 当步长选得过 大时, 它在反复递推运算中引入的误差积累将越来越大, 使计算结果出现不稳定, 本来稳定的系 统可能出现不稳定。而实时仿真必须允许采用大步长, 因而有必要对实时 RK 公式的稳定域进行 分析。实时 $s$ 级 $p$ 阶 RK 公式一般形式为

$$
\left\{\begin{array}{l}
y_{n+1}=y_{n}+h \sum_{i=1}^{s} c_{i} k_{i} \\
k_{i}=f\left[t_{n}+a_{i} h, y_{n}+h \sum_{j=1}^{i} b_{i j} k_{j}, u\left(t_{n}+a_{i} h\right)\right] \\
a_{i}=\sum_{j=1}^{i} b_{i j}, \quad i=1,2, \ldots, s
\end{array}\right.
$$

为了采样方便, 一般输入采样区间为 $\Delta t=h / s$, 则 $a_{i}=0,1 / s, 2 / s, \cdots, p / s$ 。当 $s=p+1$ 时, 相应的稳定函数为 ${ }^{[5]}$

$$
P(z)=1+z+\frac{1}{2 !} z^{2}+\cdots+\frac{1}{p !} z^{p}+c_{p+1} \prod_{i=1}^{p} b_{i+1, i} z^{p+1}
$$


据此, 在复平面上的绝对稳定区域定义为

$D=\{z:|P(z)|<1\}$

令

$\alpha_{1}=c_{p+1} \prod_{i=1}^{p} b_{i+1, i}, \quad z=\rho e^{j \theta}$

则

$P(z)=P\left(\rho, \theta ; \alpha_{1}\right)$

由于 $P(z)$ 是关于实轴对称的函数和 $\operatorname{Re} z \leq 0$, 一般选择优化目标函数及约束为

$$
Q(\alpha)=\max _{\alpha} \int_{\pi / 2}^{\pi}\left|P\left(\rho, \theta ; \alpha_{1}\right)\right| \mathrm{d} \theta
$$

$\left\{\begin{array}{l}\rho \geq 0 \\ \left|P\left(\rho, \theta ; \alpha_{1}\right)\right|<1\end{array}\right.$

由于绝对稳定域面积的大小决定 RK 公式的稳定性, 而且关于绝对稳定域面积的最大值求解 是一个典型的数值优化求解问题, 因而适于采用遗传算法求解此类约束优化问题来确定 RK 公式 的稳定性。对于目标函数最大化问题, 可用其负值的最小化问题替代为目标函数标准形式, 求解 后, 将结果再反号, 则为原目标函数的最大化值。另外约束为 $g_{i}\left(x_{1}, \cdots, x_{j, \cdots, x_{n}}\right) \geq 0$ 的形式, 可转换为 $-g_{i}\left(x_{1}, \cdots, x_{j, \cdots, x_{n}}\right) \leq 0$ 的约束标准形式 ${ }^{[6]}$ 。据此, 实时 $\mathrm{RK}$ 公式稳定域优化目标函数及约束标准形式 为:

$$
\begin{aligned}
& Q(\alpha)=\min _{\alpha} \int_{\pi / 2}^{\pi}-\left|P\left(\rho, \theta ; \alpha_{1}\right)\right| \mathrm{d} \theta \\
& \left\{\begin{array}{l}
-\rho \leq 0 \\
\left|P\left(\rho, \theta ; \alpha_{1}\right)\right|-1<0
\end{array}\right.
\end{aligned}
$$

定义群体的个体数为 35 , 最大迭代次数为 200 , 交叉概率为 0.97 , 变异概率为 0.04 , 求得实 时 RK 公式最优参数值和对应的目标函数值为表 1 所示。

表 1 实时 RK 公式的最大绝对稳定域面积

\begin{tabular}{cccc}
\hline 实时 RK 公式 & 三级二阶 & 四级三阶 & 五级四阶 \\
\hline$\alpha_{1}$ & 0.07174 & 0.020854 & 0.004494 \\
稳定域面积 & 8.43563 & 9.75567 & 11.37342 \\
\hline
\end{tabular}

\section{2 实时 RK 公式截断误差参数优化}

在确定实时 RK 公式的最大绝对稳定域后, 截断误差则是构造实时 RK 公式的另一重要指标。 
由 RK 公式的局部截断误差定义可以推导出 (2) 式的局部截断误差 ${ }^{[7]}$ 为关于 $\zeta$ 的函数 $E$

其中,

$$
\zeta=\left[\zeta_{1}, \zeta_{2}, \cdots, \zeta_{k}\right]^{T}
$$

而且 $\zeta$ 是关于 (2) 式中各系数 $c_{i}, a_{i}, b_{i j}$ 的函数, 而这些系数又必须满足一定的阶条件方程。阶 条件方程也是关于系数 $c_{i}, a_{i}, b_{i j}$ 的非线性方程, 再加上前面的稳定域寻优结果方程

$$
\alpha_{1}=c_{p+1} \prod_{i=1}^{p} b_{i+1, i}
$$

于是, 我们得到了一个包含多个参数、多个代数方程的非线性方程组。选择 $c_{i}$ 和 $b_{i j}$ 作为自由参数, 则其它相关参数都是它们的函数。取局部截断误差函数

$$
B=B(\zeta)=\sum_{i=1}^{k}\left|\zeta_{i}\right|
$$

则 $B$ 为 $c_{i}$ 和 $b_{i j}$ 的函数, 即有

$$
B=B(\zeta)=B\left(c_{i}, b_{i j}\right)
$$

选择优化目标函数为

$$
B\left(c_{i}, b_{i j}\right)=\min _{c_{i}, b_{i j}} \sum_{i=1}^{k}\left|\zeta_{i}\right|
$$

约束为上述的非线性方程组。最后再次将目标函数和约束转换为标准形式, 将优化目标函数及约 束采用遗传算法进行寻优计算, 求解出截断误差参数为表 2 所示。

表 2 实时 RK 公式截断误差参数

\begin{tabular}{cccc}
\hline 实时 RK 公式 & 三级二阶 & 四级三阶 & 五级四阶 \\
\hline 截断误差参数 & 0.095238 & 0.0140736 & 0.0072357
\end{tabular}

\section{3 经过优化的新实时 RK 公式}

通过以上基于遗传算法的实时 $\mathrm{RK}$ 公式参数优化, 可计算出实时 $\mathrm{RK}$ 公式中 $c_{i}$ 和 $b_{i j}$ 参数具体 数值。最后可推导出经过参数优化后的实时三级二阶 RK 公式、实时四级三阶 RK 公式、实时五级 四阶 RK 公式 ${ }^{[8]}$ 分别为

$$
\left\{\begin{aligned}
y_{n+1} & =y_{n}+h\left(2.49689 k_{1}-4.49378 k_{2}+2.99689 k_{3}\right) \\
k_{1} & =f\left[t_{n}, y_{n}\right] \\
k_{2} & =f\left[t_{n}+\frac{1}{3} h, y_{n}+\frac{1}{3} k_{1}\right] \\
k_{3} & =f\left[t_{n}+\frac{2}{3} h, y_{n}+h\left(0.59516 k_{1}+0.0715 k_{2}\right)\right]
\end{aligned}\right.
$$




$$
\begin{aligned}
& \left\{\begin{aligned}
y_{n+1} & =y_{n}+h\left(0.1526 k_{1}+0.20886 k_{2}+0.12447 k_{3}+0.51406 k_{4}\right) \\
k_{1} & =f\left[t_{n}, y_{n}\right] \\
k_{2} & =f\left[t_{n}+\frac{1}{4} h, y_{n}+\frac{1}{4} k_{1}\right] \\
k_{3} & =f\left[t_{n}+\frac{2}{4} h, y_{n}+h\left(0.30797 k_{1}+0.19203 k_{2}\right)\right] \\
k_{4} & =f\left[t_{n}+\frac{3}{4} h, y_{n}+h\left(0.34384 k_{1}-0.43803 k_{2}+0.8442 k_{3}\right)\right]
\end{aligned}\right. \\
& \left\{\begin{aligned}
y_{n+1} & =y_{n}+h\left(0.1174 k_{1}-0.0112 k_{2}+0.746 k_{3}-0.4279 k_{4}+0.5757 k_{5}\right) \\
k_{1} & =f\left[t_{n}, y_{n}\right] \\
k_{2} & =f\left[t_{n}+\frac{1}{5} h, y_{n}+\frac{1}{5} h k_{1}\right] \\
k_{3} & =f\left[t_{n}+\frac{2}{5} h, y_{n}-0.00676 h k_{1}+0.40676 h k_{2}\right] \\
k_{4} & =f\left[t_{n}+\frac{3}{5} h, y_{n}+0.01628 h k_{1}+0.236 h k_{2}+0.34772 h k_{3}\right] \\
k_{5} & =f\left[t_{n}+\frac{4}{5} h, y_{n}+0.2554 h k_{1}-0.24749 h k_{2}+0.51613 h k_{3}+0.27597 h k_{4}\right]
\end{aligned}\right.
\end{aligned}
$$

\section{4 结语}

遗传算法寻优简单、鲁棒性强、易于并行化，是一种高效的全局寻优方法。遗传算法求解约 束优化问题是 GA 研究中的热门问题之一。本文所涉及的实时 RK 公式参数优化模型是一种典型的 约束优化问题, 为保证遗传算法的高效高速寻优, 在对实时 RK 公式参数优化时, 必须选定合适 的优化目标, 确定约束条件。通过仿真实例可以证明, 基于遗传算法的实时 RK 公式参数优化所 得新实时 RK 公式相对经典 RK 公式具有更广泛的应用价值。

\section{5 致谢}

基金项目：广西自然科学基金项目（2013GXNSFAA019021）

\section{Acknowledgement}

Fund Project: Guangxi Natural Science Foundation Project (2013GXNSFAA019021)

\section{参考文献}

[1] 自适应粒子群优化 [C]. “系统-人-控制论” 电气与电子工程师协会, (B 部分) 控制论, 2009-39（6)： 1362 - 1381.

[2]褚胜国, 俞望. 基于遗传算法的液压伺服系统管路及仪表布置图参数优化 $[J]$. 机床液压, 2004（ 8): 14-16.

[3] Lambert J D. 常微分方程中的计算方法 [M]. 纽约: 约翰威利出版社, 1973.

[4]敖友媛, 迟洪钦. 用于解决约束函数优化问题的遗传算法 $[J]$. 燕山大学学报, 2005, 29（4）：294-298.

[5] Tanaka M. 具有误差估计能力的 Runge-Kutta 公式[J]. 可重复性统计应用研究, 日本科学家和工程师联 合会, 1966, $13(3)$ : 42-61.

[6]汇民，黄振权. 具有最大稳定性区域的实时 Rk 公式[J]. 系统仿真学报, 2006，18(2)：306-308. 


\section{References}

[1] Adaptive Particle Swarm Optimization. IEEE Transactions on Systems, Man, and Cybernetics, Part B: Cybernetics. 2009, 39 (6): 1362-1381.

[2] Chusheng guo, yu wang. Optimization of PID Parameter for Hydraulic Servo System Based On Genetic Algorithm [J]. Machine Tool \& Hydraulic, 2004, 8: 14-16.

[3] Lambert J D. Computational methods in ordinary differential equations [M]. Wiley, New York, 1973.

[4] AO You-Yuan, Chi Hong-Qin. A Genetic Algorithm for Solving Constrained Function Optimization Problems [J]. Journal of Yanshan University, 2005, 29(4): 294-298.

[5] Tanaka M. Runge-Kutta formulas with the ability of error estimation [J]. Rep. Stat. Appl. Res. JUSE, 1966, 13(3): 42-61.

[6] Jiang Min, Huang Zhen-Quan. Real-Time Rk Formula with Maximum Stability Region [J]. Journal of System Simulation, 2006, 18(2): 306-308. 